\title{
Determining the Score for Depression and Its Relationship with the Level of Physical Activity in a Patient at the Family Medicine
}

\author{
Zivana Gavric \\ Department of Social Medicine, Medical Faculty, University of Banja Luka, Banja Luka, Bosnia-Herzegovina \\ Email: higija@inecco.net
}

Received June $26^{\text {th }}, 2012$; revised July $29^{\text {th }}, 2012$; accepted August $10^{\text {th }}, 2012$

\begin{abstract}
Introduction: Drop in levels of physical activity is associated with occurrence of diseases that can lead to disability and death. Objective: To determine the level of physical activity and the presence of depression, as well their relationship with body mass index in patients. Methods: In the family medicine clinic, within the pilot study, in the first half of 2010 year, through the Interview with 141 patients, the following questionnaires were completed: International Physical Activity Questionnaire (IPAQ) and Patient Health Questionnaire (PHQ-9). The data were processed according to the instructions for questionnaires for PHQ-9 and IPAQ. The patients underwent measurements of blood pressure, sugar and cholesterol levels in blood and body mass index (BMI). Results: Of the total 141 respondents, more than half (61.70\%) were aged $25-54$ years, median 44 th years $( \pm 14.3)$. Nearly $3 / 4$ of respondents $(73.76 \%)$ had the PHQ-9 score value of $\leq 4$, and slightly more than $1 / 4$ of the respondents had the PHQ- 9 score value of $\geq 5$. The largest number of respondents $(51.80 \%)$ over the week had moderate physical activity, with statistically significant difference $\left(\chi^{2}=566.7, \mathrm{p}<0.01\right)$ compared to those who had vigorous $(16.30 \%)$ and low $(32.00 \%)$ physical activity. Over $2 / 3$ of respondents $(67.60 \%)$ with the PHQ- 9 score $\geq 5$ had high BMI and were obese and engaged in moderate physical activity (48.00\%). The median sitting in minutes in the last week was greater in those with PHQ-9 score $\geq 5$ (545 minutes) compared to those with PHQ-9 score $\leq$ 4. Discussion: Moderate physical activity is important in preventing depression and stress. Conclusion: Obligation of the family doctor is to promote physical activity in all age groups as an important factor for improving health and preventing disease.
\end{abstract}

Keywords: Physical Activity; Depression; PHQ-9 Score; The IPAQ Value

\section{Introduction}

Besides the leading risk factors for health such as smoking, poor nutrition, increasing cholesterol and blood sugar, hypertension, physical inactivity and stress, nowadays the occurrence of depression is very common.

It is recognized that the growing epidemics of obesity is associated with decline in physical activity levels and depression. Current assessment of World Health Organization (WHO, 2007) in 2007 report is that $60 \%$ to $85 \%$ of adults worldwide "are not active enough to benefit their health." Accurate assessment of physical activity is essential for planning, implementing, and evaluating public health programs and policies addressing this issue (Barnett, 2007). The physical and psychological benefits of physical activity are well documented and are highlighted in the Chief Medical Officer's report which recommends at least 30 minutes of moderate intensity physical activity a day (Donaldson, 2007). A lack of physical activity is the major factor contributing to diverse diseases, disability and mortality. Preliminary studies of WHO related to risk factors report that sedentary life style is one of ten leading causes of death and disability (WHO, 2002).

The International Physical Activity Questionnaire (IPAQ, 2007) was developed as a set of internationally comparable, valid, and reliable instruments (Craig, Marshall, Sjostrom et al., 2003) that examine self-reported Physical Activity and sedentary behaviors (Guidelines for IPAQ, 2005).

The role of primary health care is essential in promoting healthy lifestyles and physical activity in people of all ages and in the prevention of chronic diseases which we encounter more and more nowadays. Physical inactivity is commonly associated with depression. Depression is marked as a leading cause of disability and premature death in persons aged $18-44$ years worldwide and is expected to be the second leading cause of disability in persons of all age until 2020 (WHO, 2002; Haskell, Lee, Pate et al., 2007).

Family doctors in primary care have more opportunity to see a patient with depression than with any other disorder, except with hypertension. Patient Health Questionnaire (PHQ-9) is an excellent screening tool for depression in primary health care (Kroenke, Spitzer, \& Williams, 2001; Dietrich, Oxaman, Burns et al., 2003; Pinto-Meza, Serrano-Blanco, Penarrubia et al., 2005).

Research indicates that regular exercise can reduce depressive symptoms. There is, however, a need for more clinical trials on depression and physical activity to be conducted before exercise can be recommended as an alternative to more traditional pharmacological and behavioral therapies (Brosse, Sheets, Lett, \& Blumenthal, 2002).

There is also evidence to suggest that exercise has antidepressant and anxiolytic effects that protect individuals against the harmful consequences of stress (Salmon, 2001).

Babyaket et al. (Babyaket et al., 2000) in their study assessed the status of 156 adult volunteers with major depressive disorder (MDD) 6 months after completion of the study who were randomly assigned a 4-month course of aerobic exercise, ser- 
traline therapy, or a combination of exercise and sertraline. The presence and severity of depression were assessed by clinical interview using the Diagnostic Interview Schedule and the Hamilton Rating Scale for Depression (HRSD) and by self-report using the Beck Depression Inventory. Assessments were performed at baseline, after 4 months of treatment, and it was concluded 6 months after the treatment (i.e., after 10 months). Four months later the patients in all three groups exhibited significant improvement; the proportion of remitted participants (i.e., those who no longer met diagnostic criteria for MDD and had an HRSD score $<8$ ) was comparable across the three treatment conditions. After 10 months, however, remitted subjects in the exercise group had significantly lower relapse rates than subjects in the medication group. Exercising on one's own during the follow-up period was associated with a reduced probability of depression diagnosis at the end of that period (Ainsworth et al., 2000).

In cross-sectional studies it was found that there is a link between habits to protection from harmful effects of stress and physical and mental health, but causality is not clear. Nevertheless, the pattern of evidence suggests the theory that exercise training recruits a process which confers enduring resilience to stress. This view allows the effects of exercise to be understood in terms of existing psychobiological knowledge, and it can thereby provide the theoretical base that is needed to guide future research in this area. Clinically, exercise training continues to offer clinical psychologists a vehicle for nonspecific therapeutic social and psychological processes. It also offers a specific psychological treatment that may be particularly effective for patients for whom more conventional psychological interventions are less acceptable (Salmon, 2001).

Sufficient evidence now exists for the effectiveness of exercise in the treatment of clinical depression. Additionally, exercise has reducing anxiety and can improve physical self-perceptions and in some cases global self-esteem. Also there is good evidence that aerobic and resistance exercise enhances mood states, and weaker evidence that exercise can improve cognitive function (primarily assessed by reaction time) in older adults. The research suggests that moderate regular exercise should be considered as a viable means of treating depression and anxiety and improving mental well-being in the general public. In the last 15 years there has been increasing research into the role of exercise in the treatment of mental health, and in improving mental well-being in the general population. There are now several hundred studies and over 30 narrative or meta-analytic reviews of research in this field. These have summarized the potential for exercise as a therapy for clinical or subclinical depression or anxiety, and the use of physical activity as a means of upgrading life quality through enhanced self-esteem, improved mood states, reduced state and trait anxiety, resilience to stress, or improved sleep. Physical activity can be viewed from four different perspectives and it is regarding its direct contribution to solving mental health problems: Treatment of mental illness and disorders; prevention of mental illness and disorders; improvement of mental and physical well-being of those with mental illness; and improvement of mental well-being of the general population. Exercise should be promoted regardless of its impact on mental health as it carries significant reduction in risks for a range of diseases and disorders for all sectors of society. The physical activities could be a cheap and available vehicle for improving self-perceptions, mood, life satisfaction, social interaction and quality of life. There is also sufficient evidence to support the use of exercise in the treatment of depression, anxiety and stress. Treatment interventions should be tried and tested for cost effectiveness in field settings. There is no definitive exercise recommendation for all elements of mental health promotion as it is likely that different formulas of frequency, intensity and duration of exercise apply for different mechanisms and perhaps different populations. However, the current general message of 30 minutes or more of moderate activity such as brisk walking 5 or 6 days per week is supported by literature (Fox, 1999).

\section{Objective}

- To establish the level of physical activity among patients in family medicine clinics.

- To establish the existence of depression symptom among patients in family medicine clinics.

- To determine relationship between the level of physical activity and PHQ-9 score for depression with body mass index (BMI) in family medicine patients.

\section{Hypotheses}

- The level of physical activity is low in patients in family medicine clinics.

- Symptoms of depression are present in patients in family medicine clinics.

- The level of physical activity does not affect PHQ-9 score for depression and body mass index (BMI) in patients in the family medicine.

\section{Methods}

In Family Medicine Clinic in Banjaluka, in the first half of 2010, we have conducted a pilot study with 141 patients who filled the questionnaires: IPAQ short form and PHQ-9 interviews were conducted with patients who visited the family doctor for examination. The patients underwent measurements of blood pressure (systolic and diastolic), sugar and cholesterol levels in blood and body mass index (BMI).

Criteria for inclusion in the study were: age $\geq 18$ years and informed consent. Criteria for exclusion from the study were: younger than 18 years, the existence of former mental disorders and diseases that may limit physical activity.

Collected data were prepared and processed according to the instructions given for the IPAQ questionnaire (Haskell, Lee, Pate et al., 2007; Craig, Marshall, Sjostrom et al., 2003; Guidelines for IPAQ, 2005) and PHQ-9 (Kroenke, Spitzer, \& Williams, 2001). The PHQ-9) developed by Pfizer Inc. has proven to be an excellent means of monitoring levels of severity of depression over time. During the course of monitoring patient adherence to depression treatment plans, care managers utilize a variety of forms that both assist in the collection and summarizing of information about individual patient status. These forms also provide an essential means of coordinating communication for care managers and clinicians.

IPAQ is used for physical activity measurement and it is designed to assess the level of physical activity for persons 18 69 years old (Haskell, Lee, Pate et al., 2007). Both categorical and continuous indicators of physical activity are possible from both IPAQ forms. However, given the abnormal distribution of 
energy expenditure in many populations, it is suggested that the continuous indicator be presented as median minutes/week or median (metabolic equivalent tasks) MET-minutes/week rather than means (such as mean minutes/week or mean MET-minutes/week). The IPAQ Research Committee proposes that these data are reported as comparisons of median values and inter quartile ranges for different populations. The three levels of physical activity were proposed for the population and they were classified as: Low, Moderate and High (Vigorous) (Haskell, Lee, Pate et al., 2007).

The selected MET values were derived from work undertaken during the IPAQ Reliability Study undertaken in 20002001 (Guidelines for IPAQ, 2005). Using the Ainsworth et al. (Ainsworth et al., 2000) Compendium (Bucksch, 2005) an average MET score was derived for each type of activity.

Statistical analysis included descriptive statistics. For statistical data analysis, the following statistical parameters were used: frequency, percentage, score, and $\chi^{2}$-test with statistical significance level of $95 \%$. For data processing we used SPSS software program.

\section{Results}

Nearly 3/4 of respondents $(73.76 \%$ ) had the PHQ-9 score value of $\leq 4$, and slightly more than $1 / 4(26.24 \%)$ of the respondents had the PHQ-9 score value of $\geq 5$. Of the total 141 respondents, more than half $(61.70 \%)$ were aged 25 - 54 years and almost $1 / 4(23.40 \%)$ aged $55-65$ years. Almost two thirds of respondents $(62.10 \%)$ with PHQ-9 score of $\geq 5$ were aged 25 - 54 years with a statistically significant difference compared to other age groups $\left(\chi^{2}=669.288, p<0.01\right)$. Of the total number of respondents in the highest percentage were represented the patients who were employed $(51.80 \%)$ and pensioners $(25.50 \%)$ compared to the unemployed and students, with statistically significant difference $\left(\chi^{2}=552.07, \mathrm{p}<0.01\right)$ in the patients with PHQ-9 score $\leq 4$ and with PHQ-9 score of $\geq 5\left(\chi^{2}=\right.$ $227.4756, \mathrm{p}<0.01)$. With respect to sex there were more women $(52.48 \%)$ than men $(47.52 \%)$ in the sample (Table 1).

Respondents were aged 18 - 69 years with median 44th years $( \pm 14.3)$, systolic blood pressure from 90 to $170 \mathrm{mmHg}$ with the mean value $126.65( \pm 17.28)$, while diastolic pressure was from 60 to $100 \mathrm{mmHg}$ with a mean value of $98.53( \pm 9.75)$. The blood glucose levels ranged from 3.2 to 16.96 with a mean value of $5.44 \mathrm{mmol}( \pm 1.39)$ and the levels of cholesterol from 3.4 to 8.62 with a mean value of $5.39 \mathrm{mmol} / 1( \pm 1.11)$, while median BMI was $26.91 \mathrm{~kg} / \mathrm{m}( \pm 4.31)$ (Table 2).

The highest percentage of respondents were engaged in moderate physical activity $(51.80 \%)$ with statistically significant difference $\left(\chi^{2}=566.7, \mathrm{p}<0.01\right)$ compared to those who were engaged in vigorous $(16.30 \%)$ and low $(32.00 \%)$ physical activity. With respect to the value of PHQ-9 score and with PHQ9 score $\leq 4$ and with the PHQ- 9 score $\geq 5$ the highest percentage was engaged in moderate physical activity (Table 3 ).

Patients with PHQ-9 score $\leq 4$ in the highest percentage were

Table 1.

Distribution of the sample by age, profession and sex in relation to the PHQ-9 scores.

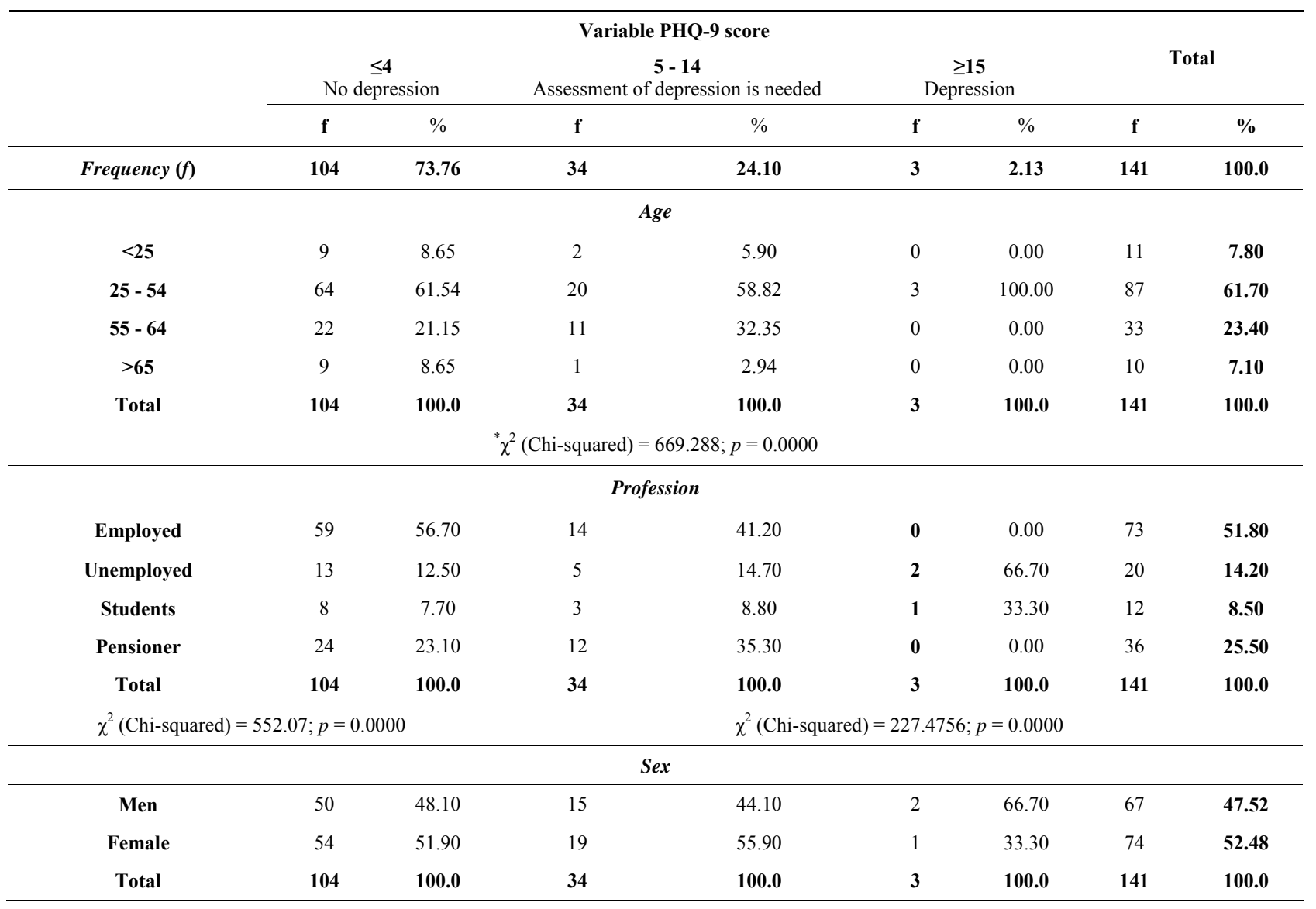


Table 2.

The variables by age, systolic and diastolic blood pressure, body mass index, levels of cholesterol and sugar in blood of the patients.

\begin{tabular}{|c|c|c|c|c|c|c|}
\hline \multirow{2}{*}{ Variables } & \multirow{2}{*}{ Age } & \multicolumn{2}{|c|}{ Blood pressure } & \multirow{2}{*}{ Body mass index-BMI $\left(\mathrm{kg} / \mathrm{m}^{2}\right)$} & \multicolumn{2}{|c|}{ In blood } \\
\hline & & Systolic (mmHg) & Diastolic (mmHg) & & Sugar $(\mathrm{mmol} / \mathrm{l})$ & Cholesterol (mmol/l) \\
\hline Min & 18 & 90 & 60 & 17.59 & 3.2 & 3.4 \\
\hline Max & 69 & 170 & 100 & 38.10 & 16.96 & 8.62 \\
\hline Me & 44 & 126.65 & 98.53 & 26.91 & 5.44 & 5.39 \\
\hline SD & 14.3 & 17.28 & 9.75 & 4.31 & 1.39 & 1.11 \\
\hline
\end{tabular}

Table 3.

The scores PHQ-9 compared to the IPAQ variable in the patients.

\begin{tabular}{|c|c|c|c|c|}
\hline \multirow{2}{*}{\multicolumn{2}{|c|}{ Variable IPAQ }} & \multicolumn{2}{|c|}{ Variable PHQ-9 score } & \multirow[b]{2}{*}{ Total } \\
\hline & & \multirow{2}{*}{$\begin{array}{c}\begin{array}{c}\leq 4^{*} \\
\text { No depression }\end{array} \\
14\end{array}$} & \multirow{2}{*}{$\begin{array}{c}\begin{array}{c}\geq \mathbf{5}^{*} \\
\text { Assessment of depression is needed }\end{array} \\
9\end{array}$} & \\
\hline \multirow{3}{*}{ Low } & $\mathrm{f}$ & & & 23 \\
\hline & $\%$ & 13.50 & 24.30 & 16.30 \\
\hline & CI & $7.6-21.6$ & $10.7-67.6$ & $12.9-24.6$ \\
\hline \multirow{3}{*}{ Moderate $^{*}$} & $\mathrm{f}$ & 55 & 18 & 73 \\
\hline & $\%$ & 52.90 & 48.60 & 51.80 \\
\hline & $\mathrm{CI}$ & $42.8-62.8$ & $32.4-67.6$ & $43.3-58.3$ \\
\hline \multirow{3}{*}{ Vigorous } & $\mathrm{f}$ & 35 & 10 & 45 \\
\hline & $\%$ & 33.7 & 27.00 & 32.00 \\
\hline & CI & $24.7-43.6$ & $12.9-44.4$ & $29.3-38.2$ \\
\hline \multirow{2}{*}{ Total } & $\mathrm{f}$ & 104 & 37 & 141 \\
\hline & $\%$ & 100 & 100 & 100 \\
\hline
\end{tabular}

${ }^{*} \chi^{2}=566.7 ; p=0.0000$.

dealing with moderate physical activity $(52.90 \%)$ regardless of the value of BMI with statistically significant differences $\left(\chi^{2}=\right.$ $419.396, \mathrm{p}<0.01)$ compared to those with vigorous $(33.70 \%)$ and low physical activity $(13.50 \%)$. More than half of the patients $(54.30 \%)$ with normal BMI were engaged in moderate and $31.40 \%$ in vigorous physical activity. Almost two thirds of patients (64.40\%) with PHQ-9 score $\leq 4$ had elevated BMI values and were obese and engaged in moderate physical activity $(50.70 \%)$.

Almost half of the patients (48.65\%) with PHQ-9 score $\geq 5$ were engaged in moderate physical activity regardless of the BMI value with statistically significant difference $\left(\chi^{2}=140.958\right.$, $\mathrm{p}<0.01)$ compared to those who were engaged in vigorous $(27$, $03 \%$ ) and those engaged in low physical activity (24.32\%). Over two thirds $(67.60 \%)$ with the PHQ-9 score $\geq 5$ had elevated BMI values and were overweight and engaged in moderate $(48.00 \%)$ and vigorous (36.00\%) physical activity (Table 4).

Sitting mean value in minutes during the last week show that these values were associated with the PHQ-9 scores $\left(r^{2}=0.87\right)$, and the patients with PHQ-9 score $\geq 5$ (545 minutes) were sitting more during the week as compared to those with PHQ-9 score $\leq 4$ (459 minutes) (Figure 1).

\section{Discussion}

The study about relation between physical activity and mental health was conducted in 15 countries of European Union and included 16230 persons. The conclusion of the study was that those who are physically more active are in general of better mental health (Richard, Kristin, \& Vickers, 2006).

In our sample about half of the patients were physically active in a form of moderate physical activity.

The importance of physical activity as a therapy for clinical or sub clinical depression or anxiety and improvement of life quality through enhanced self-esteem, improved mood, reduced stress and anxiety, resilience to stress, or improved sleep was reported in the study conducted in the UK. Regular moderate training should be considered an important part of treatment of depression and anxiety, as well as improving mental health in general population (Fox, 1999).

Richard et al. conducted a meta-analysis of exercise as a treatment for depression. The empirical evidence is convincing that exercise either alone or in combination with other evidence-supported treatment is effective in treating clinically significant depression. The challenge lies in translating the convincing evidence into effective practice. This commentary 
Table 4.

Variables IPAQ in relation to BMI in the patients with PHQ-9 score $\leq 4$ and PHQ-9 score $\geq 5$.

\begin{tabular}{|c|c|c|c|c|c|c|c|c|}
\hline \multicolumn{9}{|c|}{ PHQ-9 score $\leq 4$ (No depression) } \\
\hline \multirow{3}{*}{ Body mass index-BMI } & \multicolumn{6}{|c|}{ Variable IPAQ } & \multirow{2}{*}{\multicolumn{2}{|c|}{ Total }} \\
\hline & \multicolumn{2}{|c|}{ Low } & \multicolumn{2}{|c|}{ Moderate } & \multicolumn{2}{|c|}{ Vigorous } & & \\
\hline & f & $\%$ & f & $\%$ & f & $\%$ & f & $\%$ \\
\hline$<18.5$ & 1 & 50.00 & 1 & 50.00 & 0 & 0.00 & 2 & 100.0 \\
\hline $18.5-25.6$ & 5 & 14.30 & 19 & 54.30 & 11 & 31.40 & 35 & 100.0 \\
\hline $25.7-30.0$ & 4 & 10.00 & 22 & 55.00 & 14 & 35.00 & 40 & 100.0 \\
\hline$>\mathbf{3 0}$ & 4 & 14.80 & 13 & 48.10 & 10 & 37.00 & 27 & 100.0 \\
\hline Total & 14 & 13.50 & 55 & 52.90 & 35 & 33.70 & 104 & 100.0 \\
\hline & & & & 19.396 & & & & \\
\hline
\end{tabular}

PHQ-9 score $\geq \mathbf{5}$ (Assessment of depression is needed)

\begin{tabular}{|c|c|c|c|c|c|c|c|c|}
\hline \multicolumn{9}{|c|}{ Body mass index-BMI } \\
\hline$<18.5$ & 0 & 0.0 & 0 & 0.0 & 0 & 0.0 & 0 & 0.0 \\
\hline $18.5-25.6$ & 5 & 41.70 & 6 & 50.00 & 1 & 8.30 & 12 & 100.0 \\
\hline $25.7-30.0$ & 3 & 16.67 & 8 & 44.43 & 7 & 38.90 & 18 & 100.0 \\
\hline$>\mathbf{3 0}$ & 1 & 14.30 & 4 & 57.10 & 2 & 28.60 & 7 & 100.0 \\
\hline Total & 9 & 24.32 & 18 & 48.65 & 10 & 27.03 & 37 & 100.0 \\
\hline \multicolumn{9}{|c|}{$\chi^{2}($ Chi-squared $)=140.958 ; p=0.0000$} \\
\hline
\end{tabular}

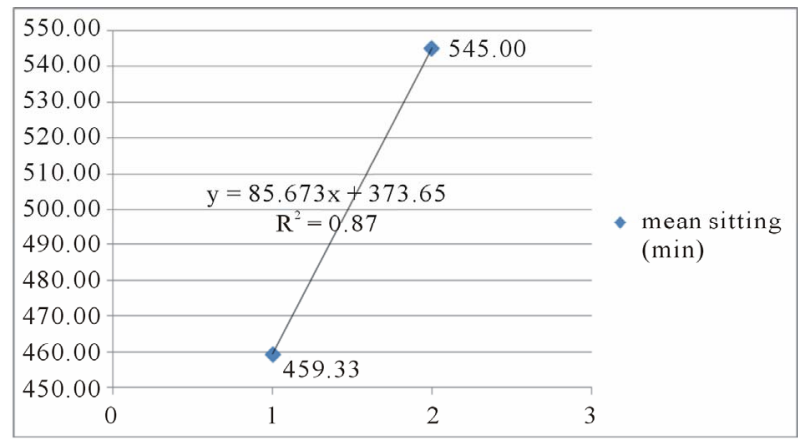

Figure 1.

Correlation between average level seating and PHQ-9 score. ${ }^{*} 1=$ PHQ9 for PHQ-9 score $\leq 4 ;{ }^{*} 2$ = PHQ-9 for PHQ-9 score $\geq 5$.

focuses on the barriers that clinicians encounter in utilizing exercise in the face of depressive symptoms, pessimism, low motivation, and physical inactivity and withdrawal. Also they discuss some practical suggestions to enhance the likelihood that patients with depression implement and maintain exercise behaviors to improve their mood (Richard, Kristin, \& Vickers, 2006).

In a study at the University of Illinois the effects of 2 physical activity modes, walking and low-intensity resistance/flexibility training were examined, on depressive symptoms and physical self-esteem over a 5-year period among older adults. Results suggest that older adults who participate in walking or low-intensity resistance/flexibility training experience sustained reductions in depression symptoms and increases in physical self-esteem (James et al., 1999).

The study in Japan that included 575 persons age 55 - 75 years had shown that $80 \%$ of them participated in weekly physical activity and the total physical activity was similar between male and female (Fumi, Andy, \& Colin, 2008).

The study in Finland with 2840 persons followed-up the incidence of $\mathrm{CAD}$, hypertension and diabetes during 10-years period had shown that vigorous activity once or more often per week was inversely associated with the risk of hypertension, both in men and women. For the women both a higher total amount of activity and weekly vigorous activity had an inverse association with the risk of diabetes (Haapanen, Miilunpalo, Vuori, Oja, \& Pasanen, 1997).

The meta-analysis which was performed by systematic MEDLINE search in 2007 included 33 studies with 883372 persons, the follow-up range from 4 to 20 years. Majority of studies reported significant risk reduction for physical active participants; risk reduction considering cardiovascular mortality of $35 \%$, all cause mortality of $33 \%$. Studies that used patients' questionnaires to assess physical activity reported lower risk reductions than studies that used more objective measures of fitness (Nocon, Hiemann, Müller-Riemenschneider, Thalau, Roll, \& Willich, 2008).

Study conducted in USA had investigated how the type and intensity of physical activity takes part in incidence of CAD in men. The results showed that men who run an hour or more per week had $42 \%$ risk reduction compared with men that did not run. Men who were trained with weights for 30 minutes or 
more per week had a $23 \%$ risk reduction compared with men who did not trained with weights. Rowing for one hour or more per week was associated with an $18 \%$ risk reduction. A half an hour or more of brisk walking was associated with an $18 \%$ risk reduction (Tanasescu, Leitzmann, Rimm, Willett, Stampfer, \& $\mathrm{Hu}, 2002)$.

To assess the effectiveness of an aerobic exercise program compared with standard medication (i.e., antidepressants) for treatment of MDD in older patients, a 16-week randomized controlled trial was conducted. One hundred fifty-six men and women with MDD (age, $\geq 50$ years) were assigned randomly to a program of aerobic exercise, antidepressants (sertraline hydrochloride), or combined exercise and medication. Subjects underwent comprehensive evaluations of depression, including the presence and severity of MDD using Diagnostic and Statistical Manual of Mental Disorders, Fourth Edition criteria and Hamilton Rating Scale for Depression (HAM-D) and Beck Depression Inventory (BDI) scores before and after treatment. Secondary outcome measures included aerobic capacity, life satisfaction, self-esteem, anxiety, and dysfunctional cognition's. After 16 weeks of treatment, the groups did not differ statistically on HAM-D or BDI scores adjustment for baseline levels of depression yielded an essentially identical result. Growth curve models revealed that all groups exhibited statistically and clinically significant reductions on HAM-D and BDI scores. However, patients which were receiving medication alone exhibited the fastest initial response; among patients receiving combination therapy, those with less severe depressive symptoms initially showed a more rapid response than those with initially more severe depressive symptoms (Lindwall, Rennemark, Halling, Berglund, \& Hassmen, 2007).

The study on correlation between physical activity and lifestyle conducted in Estonia showed that $92 \%$ of interviewed female family doctors did moderate to vigorous physical activity and that the level of physical activity was not related to age, BMI, living area and time they spent sitting (Suija, Pechter, Maaroos, Kalda, Ratsep, Oona, \& Maaroos, 2010).

The research conducted on the population sample based on US women who participated in the 2005 or 2006 in National Health and Nutritional Examination Survey considered the relationship between obesity and depression, which was more prominent in women than in men. A total of 10,348 individuals of all ages were included in NHANES 2005-2006. In this survey BMI was correlated positively with probability of moderate/vigorous symptoms of depression (score $\geq 10)(\mathrm{r}=0.49, p=$ $0.03)$ and major depression $(\mathrm{r}=0.72, p<0.0001)$ on PHQ-9 (Jun \& Lan, 2010).

In our study the mean sitting value in minutes during the last week show that these values are associated with the PHQ-9 scores $\left(r^{2}=0.87\right)$, and more sitting during the week of the patients with the PHQ-9 score $\geq 5$ compared to those with PHQ-9 score $\leq 4$

In a study conducted in Korea with self-reported Patient Health Questionnaire [PHQ-9] for depression, accelerometer for amounts of physical activity, demographic information, and self-reported health status were weighted to represent population estimates. Overall prevalence of depression was $13.9 \%$ and $5.6 \%$ in mild and moderate to severe levels, respectively. Adults in depression groups spent significantly less time in both light and moderate physical activity than non-depression groups. There were no differences in sedentary and vigorous physical activity among groups. After controlling for socio-demographic factors, those at greater risk for depression were middle-aged women with self-reported poor health status with less moderate physical activity (Song, Lee, Baek, \& Miller, 2012).

Physical activity is more cost-effective than either psychopharmacological or psychotherapeutic interventions. Physical activity has the potential to simultaneously improve health and well-being and tackle mental illness. Especially it is in relation with the cardiovascular diseases and diabetes and co-morbidity problems experienced by people with mental illness. For example, persons with schizophrenia tend to die not from schizophrenia, per se, but rather from co-morbid cardiovascular problems - which may be directly improved through regular physical activity (Faulkner, 2006). Physical activity plays an important role in the recovery of mental health. Richardson et al. (Richardson, Faulkner, McDevitt, Skirinar, Hutchinson, \& Piette, 2005) add two further reasons why physical activity program should be included in psychiatric services: the opportunity for individuals with mental illness to have frequent contact with their mental health service providers and that specific mental illness barriers may be best addressed by people trained in the mental health field.

As Richardson and colleagues wrote, “...changing health behaviors can be difficult, and frequent reinforcement can play a critical role in the successful long-term adoption of regular physical activity."

\section{Conclusion}

The studies among populations in different geographical areas show that it is important to exercise as prescribed in a form of moderate physical activity for good health and prevention of depression and other diseases. A role of the family doctor is very important in counseling the patients on physical activity in order to prevent health problems. Early detection of depression and other risk factors to health with timely prevention of disease should be very important to the work of family doctors.

The family doctors should advice moderate physical activity for 30 minutes five times a week, in line with the recommendations of the American Heart Association (AHA) and the American College of Sports Medicine. Also, family doctor should be trained in many issues to monitor the risk factors for health problems including the level of physical activity of the patient, and emphasis of need for daily physical activity in order to maintain health and to reduce depression. Promoting moderate physical activity for all age groups and for all groups of patients is essential in prevention of risk factors for chronic diseases, stress and depression.

\section{REFERENCES}

WHO (2007). A European framework to promote physical activity for health. Copenhagen, World Health Organization.

Barnett, J. et al. (2007). The effect of item order on physical activity estimates using the IPAQ. Californian Journal of Health Promotion, 5, 23-29.

Donaldson, L. (2007). At least five a week: Evidence on the impact of physical activity and its relationship to health. URL (last accessed February 2007).

http://www. dh. gov. uk/Publications And Statistics/Publications/ublications Policy And Guidance/fs/en

World Health Organization (2002). Move for health, Geneva. http://www.who.int/world-health day/previous/2002/en

Craig, C. L., Marshall, A., Sjostrom, M. et al. (2003). International physical activity questionnaire: 12 country reliability and validity. Medi- 
cine and Science in Sports and Exercise, 35, 1381-1395. doi:10.1249/01.MSS.0000078924.61453.FB

International Physical Activity Questionnaire (2005). Short and long forms. URL (last revised November 2005) http:// www.ipaq.ki.se

Haskell, W. L., Lee, I. M., Pate, R. R. et al. (2007). Physical activity and public health: Updated recommendation for adults from the American College of Sports Medicine and the American Heart Association. Circulation, 116, 1081-1093. doi:10.1161/CIRCULATIONAHA.107.185649

Kroenke, K., Spitzer, R. L., \& Williams, J. B. (2001). The PHQ-9: Validity of a brief depression severity measure. Journal of General Internal Medicine, 16, 606-613.

doi:10.1046/j.1525-1497.2001.016009606.x

Dietrich, A. J., Oxman, T. E., Burns, M. R. et al. (2003). Application of a depression management office system in community practice: A demonstration. Journal of the American Board of Family Medicine, 16, 107-114. doi:10.3122/jabfm.16.2.107

Pinto-Meza, A., Serrano-Blanco, A., Penarrubia, M. T. et al. (2005). Assessing depression in primary care with the PHQ-9: Can it be carried out over the telephone? Journal of General Internal Medicine, 20, 738-742. doi:10.1111/j.1525-1497.2005.0144.x

Brosse, A., Sheets, E., Lett, H., \& Blumenthal, J. (2002). Exercise and the treatment of clinical depression in adults: Recent findings and future directions. Sports Medicine, 32, 741-760. doi: $10.2165 / 00007256-200232120-00001$

Salmon, P. (2001). Effects of physical exercise on anxiety, depression, and sensitivity to stress: A unifying theory. Clinical Psychology Review, 21, 33-61. doi:10.1016/S0272-7358(99)00032-X

Babyaket, M. et al. (2000). Exercise treatment for major depression: Maintenance of therapeutic benefit at 10 months. Psychosomatic Medicine, 62, 633-638.

Ainsworth, B. E. et al. (2000). Compendium of physical activities: An update of activity codes and MET intensities. Medicine \& Science in Sports \& Exercise, 32, S498-S504.

doi:10.1097/00005768-200009001-00009

Fox, R. K. (1999). The influence of physical activity on mental wellbeing. Public Health Nutrition, 2, 411-418. doi:10.1017/S1368980099000567

Bucksch, J. (2005). Physical activity of moderate intensity in leisure time and the risk of all cause mortality. British Journal of Sports Medicine, 39, 632-638. doi:10.1136/bjsm.2004.015768

Richard, J. S., Kristin, S., \& Vickers, K. S. (2006). The challenges of treating depression with exercise: From evidence to practice. Clinical Psychology: Science and Practice, 13, 194-197. doi:10.1111/j.1468-2850.2006.00022.x

James, A. B. et al. (1999). Effects of exercise training on older patients with major depression. Archives of Internal Medicine, 159, 2349 2356. doi:10.1001/archinte.159.19.2349

Fumi, H., Andy, H. L., \& Colin, W. B. (2008). Physical activity of adults aged 55 to 75 years in Japan. Journal of Physical Therapy Science, 20, 217-220. doi:10.1589/jpts.20.217

Haapanen, N., Miilunpalo, S., Vuori, I., Oja, P., \& Pasanen, M. (1997). Association of leisure time physical activity with the risk of coronary heart disease, hypertension and diabetes in middle-aged men and women. International Journal of Epidemiology, 26, 739-747. doi:10.1093/ije/26.4.739

Nocon, M., Hiemann, T., Müller-Riemenschneider, F., Thalau, F., Roll, S., \& Willich, S. N. (2008). Association of physical activity with allcause and cardiovascular mortality: A systematic review and metaanalysis. European Journal of Preventive Cardiology, 15, 239-246. doi:10.1097/HJR.0b013e3282f55e09

Tanasescu, M., Leitzmann, M. F., Rimm, E. B., Willett, W. C., Stampfer, M. J., \& Hu, F. B. (2002). Exercise type and intensity in relation to coronary heart disease in men. JAMA, 288, 1994-2000. doi:10.1001/jama.288.16.1994.

Lindwall, M., Rennemark, M., Halling, A., Berglund, J., \& Hassmen, P. (2007). Depression and exercise in elderly men and women: Findings from the Swedish national study on aging and care. Journal of Aging and Physical Activity, 15, 41-55.

Suija, K., Pechter, U., Maaroos, J., Kalda, R., Ratsep, A., Oona, M., \& Maaroos, H. I. (2010). Physical activity of Estonian family doctors and their counseling for a healthy lifestyle: A cross-sectional study. BMC Family Practice, 11, 48.

Jun, M., \& Lan, X. (2010). Obesity and depression in US women: Results from the 2005-2006 National Health and Nutritional Examination Survey. Obesity, 18, 347-353.

Song, M. R., Lee, Y. S., Baek, J. D., \& Miller, M. (2012). Physical activity status in adults with depression in the National Health and Nutrition Examination Survey, 2005-2006. Public Health Nursing, 29, 208-217. doi:10.1111/j.1525-1446.2011.00986.x

Faulkner, G. (2006). Physical activity and mental health: A win-win consideration? PowerPoint Presentation and Lecture at the Alberta Centre for Active Living: Mental Health and Physical Activity Workshop, Banff. http://www.centre4activeliving.ca

Richardson, C. R., Faulkner, G., McDevitt, J., Skirinar, G. S., Hutchinson, D. S., \& Piette, J. D. (2005). Integrating physical activity into mental health services for persons with serious mental illness. Psychiatric Services, 56, 324-331. doi:10.1176/appi.ps.56.3.324 